\title{
Some numerical radius inequalities for power series of operators in Hilbert spaces
}

\section{Silvestru Sever Dragomir}

\begin{abstract}
By the help of power series $f(z)=\sum_{n=0}^{\infty} a_{n} z^{n}$, we can naturally construct another power series that has as coefficients the absolute values of the coefficients of $f$, namely, $f_{a}(z):=\sum_{n=0}^{\infty}\left|a_{n}\right| z^{n}$. Utilizing these functions, we show among others that

$$
w[f(T)] \leq f_{a}[w(T)]
$$

and

$$
w[f(T)] \leq \frac{1}{2}\left[f_{a}(\|T\|)+f_{a}\left(\left\|T^{2}\right\|^{1 / 2}\right)\right]
$$

where $w(T)$ denotes the numerical radius of the bounded linear operator $T$ on a complex Hilbert space, while $\|T\|$ is its norm.
\end{abstract}

MSC: 47A63; 47A99

Keywords: bounded linear operators; functions of operators; numerical radius; power series

\section{Introduction}

The numerical radius $w(T)$ of an operator $T$ on $H$ is given by $[1, \mathrm{p} .8]$

$$
w(T)=\sup \{|\lambda|, \lambda \in W(T)\}=\sup \{|\langle T x, x\rangle|,\|x\|=1\} .
$$

Obviously, by (1.1), for any $x \in H$, one has

$$
|\langle T x, x\rangle| \leq w(T)\|x\|^{2}
$$

It is well known that $w(\cdot)$ is a norm on the Banach algebra $B(H)$ of all bounded linear operators $T: H \rightarrow H$, i.e.,

(i) $w(T) \geq 0$ for any $T \in B(H)$ and $w(T)=0$ if and only if $T=0$;

(ii) $w(\lambda T)=|\lambda| w(T)$ for any $\lambda \in \mathbb{C}$ and $T \in B(H)$;

(iii) $w(T+V) \leq w(T)+w(V)$ for any $T, V \in B(H)$.

@ 2013 Dragomir; licensee Springer. This is an Open Access article distributed under the terms of the Creative Commons Attribution License (http://creativecommons.org/licenses/by/2.0), which permits unrestricted use, distribution, and reproduction in any medium, provided the original work is properly cited. 
This norm is equivalent with the operator norm. In fact, the following more precise result holds [1, p.9].

Theorem 1 (Equivalent norm) For any $T \in B(H)$, one has

$$
w(T) \leq\|T\| \leq 2 w(T) .
$$

Some improvements of (1.3) are as follows.

Theorem 2 (Kittaneh, 2003 [2]) For any operator $T \in B(H)$, we have the following refinement of the first inequality in (1.3):

$$
w(T) \leq \frac{1}{2}\left(\|T\|+\left\|T^{2}\right\|^{1 / 2}\right) .
$$

Utilizing the Cartesian decomposition for operators, Kittaneh improved the inequality (1.3) as follows.

Theorem 3 (Kittaneh, 2005 [3]) For any operator $T \in B(H)$, we have

$$
\frac{1}{4}\left\|T^{*} T+T T^{*}\right\| \leq w^{2}(T) \leq \frac{1}{2}\left\|T^{*} T+T T^{*}\right\| .
$$

From a different perspective, we have the following result as well.

Theorem 4 (Dragomir, 2007 [4]) For any operator $T \in B(H)$, we have

$$
w^{2}(T) \leq \frac{1}{2}\left[w\left(T^{2}\right)+\|T\|^{2}\right] .
$$

The following general result for the product of two operators holds [1, p.37].

Theorem 5 (Holbrook, 1969 [5]) If $A, B$ are two bounded linear operators on the Hilbert space $(H,\langle\cdot, \cdot\rangle)$, then $w(A B) \leq 4 w(A) w(B)$. In the case that $A B=B A$, then $w(A B) \leq$ $2 w(A) w(B)$. The constant 2 is best possible here.

The following results are also well known [1, p.38].

Theorem 6 (Holbrook, 1969 [5]) If A is a unitary operator that commutes with another operator $B$, then

$$
w(A B) \leq w(B) .
$$

If $A$ is an isometry and $A B=B A$, then (1.7) also holds true.

We say that $A$ and $B$ double commute if $A B=B A$ and $A B^{*}=B^{*} A$. The following result holds [1, p.38].

Theorem 7 (Holbrook, 1969 [5]) If the operators $A$ and B double commute, then

$$
w(A B) \leq w(B)\|A\| .
$$


As a consequence of the above, we have the following [1, p.39].

Corollary 1 Let A be a normal operator commuting with B. Then

$$
w(A B) \leq w(A) w(B)
$$

A related problem with the inequality (1.8) is to find the best constant $c$ for which the inequality

$$
w(A B) \leq c w(A)\|B\|
$$

holds for any two commuting operators $A, B \in B(H)$. It is known that $1.064<c<1.169$; see $[6,7]$ and [8].

Motivated by the above results, we establish in this paper some inequalities for the numerical radius of functions of operators defined by power series, which incorporate many fundamental functions of interest such as the exponential function, some trigonometric functions, the functions $f(z)=(1 \pm z)^{-1}, g(z)=\log (1 \pm z)^{-1}$ and others. Some examples of interest are also provided.

\section{Some inequalities for one operator}

Now, by the help of power series $f(z)=\sum_{n=0}^{\infty} a_{n} z^{n}$, we can naturally construct another power series which will have as coefficients the absolute values of the coefficients of the original series, namely, $f_{a}(z):=\sum_{n=0}^{\infty}\left|a_{n}\right| z^{n}$. It is obvious that this new power series will have the same radius of convergence as the original series. We also notice that if all coefficients $a_{n} \geq 0$, then $f_{a}=f$.

The following simple result provides some nice inequalities for operator functions defined by power series.

Theorem 8 Let $f(z)=\sum_{n=0}^{\infty} a_{n} z^{n}$ be a function defined by power series with complex coefficients and convergent on the open disk $D(0, R) \subset \mathbb{C}, R>0$. For any $T \in B(H)$, we have the inequality

$$
w[f(T)] \leq f_{a}[w(T)]
$$

provided $w(T)<R$, and the inequality

$$
w[f(T)] \leq \frac{1}{2}\left[f_{a}(\|T\|)+f_{a}\left(\left\|T^{2}\right\|^{1 / 2}\right)\right]
$$

provided $\|T\|<R$.

Proof Let $m \in \mathbb{N}$ with $m \geq 1$. Utilizing the properties of the numerical radius, we have

$$
w\left[\sum_{n=0}^{m} a_{n} T^{n}\right] \leq \sum_{n=0}^{m}\left|a_{n}\right| w\left(T^{n}\right) \leq \sum_{n=0}^{m}\left|a_{n}\right| w^{n}(T) .
$$


Since the series $\sum_{n=0}^{\infty}\left|a_{n}\right| w^{n}(T)$ is convergent on $\mathbb{R}$, the series $\sum_{n=0}^{\infty} a_{n} T^{n}$ is convergent in $B(H)$, and by the continuity of the numerical radius, we have

$$
\lim _{m \rightarrow \infty} w\left[\sum_{n=0}^{m} a_{n} T^{n}\right]=w\left[\sum_{n=0}^{\infty} a_{n} T^{n}\right] .
$$

Then, by letting $m \rightarrow \infty$ in the inequality (2.3), we deduce the desired result (2.1).

Utilizing the properties of the numerical radius and the Kittaneh inequality (1.4), we also have

$$
\begin{aligned}
w\left[\sum_{n=0}^{m} a_{n} T^{n}\right] & \leq \sum_{n=0}^{m}\left|a_{n}\right| w\left(T^{n}\right) \leq \frac{1}{2} \sum_{n=0}^{m}\left|a_{n}\right|\left(\left\|T^{n}\right\|+\left\|T^{2 n}\right\|^{1 / 2}\right) \\
& \leq \frac{1}{2} \sum_{n=0}^{m}\left|a_{n}\right|\left(\|T\|^{n}+\left\|T^{2}\right\|^{n / 2}\right) \\
& =\frac{1}{2}\left[\sum_{n=0}^{m}\left|a_{n}\right|\|T\|^{n}+\sum_{n=0}^{m}\left|a_{n}\right|\left(\left\|T^{2}\right\|^{1 / 2}\right)^{n}\right] .
\end{aligned}
$$

Since the series $\sum_{n=0}^{\infty}\left|a_{n}\right|\|T\|^{n}, \sum_{n=0}^{m}\left|a_{n}\right|\left(\left\|T^{2}\right\|^{1 / 2}\right)^{n}$ are convergent on $\mathbb{R}$, the series $\sum_{n=0}^{\infty} a_{n} T^{n}$ is convergent in $B(H)$. Then, by letting $m \rightarrow \infty$ in the inequality (2.4), we deduce the desired result (2.2).

Corollary 2 Let $f(z)=\sum_{n=0}^{\infty} a_{n} z^{n}$ be a function defined by power series with nonnegative coefficients and convergent on the open disk $D(0, R) \subset \mathbb{C}, R>0$. For any $T \in B(H)$, we have the inequality

$$
w[f(T)] \leq f[w(T)]
$$

if $w(T)<R$, and the inequality

$$
w[f(T)] \leq \frac{1}{2}\left[f(\|T\|)+f\left(\left\|T^{2}\right\|^{1 / 2}\right)\right]
$$

if $\|T\|<R$.

From a different perspective, we have the following.

Theorem 9 Let $f(z)=\sum_{n=0}^{\infty} a_{n} z^{n}$ be a function defined by power series with complex coefficients and convergent on the open disk $D(0, R) \subset \mathbb{C}, R>0$. For any $T \in B(H)$ with $\|T\|^{2}<R$ and $z \in \mathbb{C}$ with $|z|^{2}<r$, we have the inequality

$$
w^{2}[f(z T)] \leq \frac{1}{2} f_{a}\left(|z|^{2}\right)\left[f_{a}\left(w\left(T^{2}\right)\right)+f_{a}\left(\|T\|^{2}\right)\right]
$$

and the inequality

$$
w^{2}[f(z T)] \leq f_{a}\left(|z|^{2}\right) f_{a}\left(\left\|\frac{T^{*} T+T T^{*}}{2}\right\|\right) .
$$


Proof Let $m \in \mathbb{N}$ with $m \geq 1$. Utilizing the properties of the numerical radius, we have

$$
w^{2}\left[\sum_{n=0}^{m} a_{n} z^{n} T^{n}\right] \leq\left(\sum_{n=0}^{m}\left|a_{n}\right||z|^{n} w\left(T^{n}\right)\right)^{2} .
$$

By the weighted Cauchy-Bunyakovsky-Schwarz discrete inequality, we have

$$
\left(\sum_{n=0}^{m}\left|a_{n}\right||z|^{n} w\left(T^{n}\right)\right)^{2} \leq \sum_{n=0}^{m}\left|a_{n}\right||z|^{2 n} \sum_{n=0}^{m}\left|a_{n}\right| w^{2}\left(T^{n}\right) .
$$

Now, on writing the inequality (1.6) for the operators $T^{n}$, we have

$$
w^{2}\left(T^{n}\right) \leq \frac{1}{2}\left[w\left(T^{2 n}\right)+\left\|T^{n}\right\|^{2}\right] \leq \frac{1}{2}\left[w^{n}\left(T^{2}\right)+\|T\|^{2 n}\right]
$$

for any $n \in \mathbb{N}$, which implies that

$$
\sum_{n=0}^{m}\left|a_{n}\right| w^{2}\left(T^{n}\right) \leq \frac{1}{2}\left[\sum_{n=0}^{m}\left|a_{n}\right| w^{n}\left(T^{2}\right)+\sum_{n=0}^{m}\left|a_{n}\right|\|T\|^{2 n}\right] .
$$

On making use of the inequalities (2.9)-(2.11), we get

$$
\begin{aligned}
& w^{2}\left[\sum_{n=0}^{m} a_{n} z^{n} T^{n}\right] \\
& \quad \leq \frac{1}{2} \sum_{n=0}^{m}\left|a_{n}\right||z|^{2 n}\left[\sum_{n=0}^{m}\left|a_{n}\right| w^{n}\left(T^{2}\right)+\sum_{n=0}^{m}\left|a_{n}\right|\|T\|^{2 n}\right] .
\end{aligned}
$$

Since the series $\sum_{n=0}^{m}\left|a_{n}\right||z|^{2 n}, \sum_{n=0}^{m}\left|a_{n}\right| w^{n}\left(T^{2}\right)$ and $\sum_{n=0}^{m}\left|a_{n}\right|\|T\|^{2 n}$ are convergent on $\mathbb{R}$ and $\sum_{n=0}^{m} a_{n} z^{n} T^{n}$ is convergent on $B(H)$, then by letting $m \rightarrow \infty$ in the inequality (2.12), we deduce the desired result (2.7).

Now, on making use of the Kittaneh inequality (1.5), we also have

$$
w^{2}\left(T^{n}\right) \leq\left[w^{2}(T)\right]^{n} \leq\left\|\frac{T^{*} T+T T^{*}}{2}\right\|^{n}
$$

for any $n \in \mathbb{N}$, which implies

$$
\sum_{n=0}^{m}\left|a_{n}\right| w^{2}\left(T^{n}\right) \leq \sum_{n=0}^{m}\left|a_{n}\right|\left\|\frac{T^{*} T+T T^{*}}{2}\right\|^{n}
$$

By the inequalities (2.9) and (2.10), we then get

$$
w^{2}\left[\sum_{n=0}^{m} a_{n} z^{n} T^{n}\right] \leq \sum_{n=0}^{m}\left|a_{n}\right||z|^{2 n} \sum_{n=0}^{m}\left|a_{n}\right|\left\|\frac{T^{*} T+T T^{*}}{2}\right\|^{n}
$$

for any $m \in \mathbb{N}$ with $m \geq 1$.

The proof follows now as above and we get the desired inequality (2.8). 
Corollary 3 Let $f(z)=\sum_{n=0}^{\infty} a_{n} z^{n}$ be a function defined by power series with nonnegative coefficients and convergent on the open disk $D(0, R) \subset \mathbb{C}, R>0$. For any $T \in B(H)$ with $\|T\|^{2}<R$ and $z \in \mathbb{C}$ with $|z|^{2}<r$, we have the inequality

$$
w^{2}[f(z T)] \leq \frac{1}{2} f\left(|z|^{2}\right)\left[f\left(w\left(T^{2}\right)\right)+f\left(\|T\|^{2}\right)\right]
$$

and the inequality

$$
w^{2}[f(z T)] \leq f\left(|z|^{2}\right) f\left(\left\|\frac{T^{*} T+T T^{*}}{2}\right\|\right) .
$$

\section{Some inequalities for two operators}

We start with the following result.

Theorem 10 Let $A, B \in B(H)$ and $k>0$ such that

$$
w(A B) \leq k^{2} w(A) w(B) .
$$

If $f(z)=\sum_{n=0}^{\infty} a_{n} z^{n}$ is a function defined by power series with complex coefficients and convergent on the open disk $D(0, R) \subset \mathbb{C}, R>0$ and $k^{p} w^{p}(A), k^{q} w^{q}(B)<R$, for $p>1, \frac{1}{p}+\frac{1}{q}=1$, then we have the inequalities

$$
w(f(A B)) \leq \min \left\{M_{1}, M_{2}\right\}
$$

where

$$
M_{1}:=f_{a}^{1 / p}\left(k^{p} w^{p}(A)\right) f_{a}^{1 / q}\left(k^{q} w^{q}(B)\right)
$$

and

$$
M_{2}:=\frac{f_{a}\left(k^{p} w^{p}(A)\right) f_{a}\left(k^{q} w^{q}(B)\right)}{f_{a}\left(k^{(p+q-2)} w^{p-1}(A) w^{q-1}(B)\right)} .
$$

Proof By the properties of the numerical radius and by (3.3), we have

$$
w\left[(A B)^{n}\right] \leq w^{n}(A B) \leq k^{2 n} w^{n}(A) w^{n}(B)
$$

for any $n \in \mathbb{N}$.

Let $m \in \mathbb{N}$ with $m \geq 1$. We have, by the above inequality,

$$
w\left[\sum_{n=0}^{m} a_{n}(A B)^{n}\right] \leq \sum_{n=0}^{m}\left|a_{n}\right| w\left[(A B)^{n}\right] \leq \sum_{n=0}^{m}\left|a_{n}\right| k^{2 n} w^{n}(A) w^{n}(B) .
$$

By Hölder's weighted inequality, we have

$$
\sum_{n=0}^{m}\left|a_{n}\right| k^{2 n} w^{n}(A) w^{n}(B) \leq\left(\sum_{n=0}^{m}\left|a_{n}\right| k^{p n} w^{p n}(A)\right)^{1 / p}\left(\sum_{n=0}^{m}\left|a_{n}\right| k^{q n} w^{q n}(B)\right)^{1 / q} .
$$


Then, by (3.4) and by (3.5), we get

$$
w\left[\sum_{n=0}^{m} a_{n}(A B)^{n}\right] \leq\left(\sum_{n=0}^{m}\left|a_{n}\right| k^{p n} w^{p n}(A)\right)^{1 / p}\left(\sum_{n=0}^{m}\left|a_{n}\right| k^{q n} w^{q n}(B)\right)^{1 / q}
$$

for any $m \in \mathbb{N}$ with $m \geq 1$.

Since the series whose partial sums are involved in (3.6) are convergent, then by taking $m \rightarrow \infty$ in (3.6), we deduce the first inequality in (3.2).

Further, by utilizing the following Hölder-type inequality obtained by Dragomir and Sándor in 1990 [9] (see also [10, Corollary 2.34]):

$$
\sum_{k=0}^{n} m_{k}\left|x_{k}\right|^{p} \sum_{k=0}^{n} m_{k}\left|y_{k}\right|^{q} \geq \sum_{k=0}^{n} m_{k}\left|x_{k} y_{k}\right| \sum_{k=0}^{n} m_{k}\left|x_{k}\right|^{p-1}\left|y_{k}\right|^{q-1}
$$

that holds for nonnegative numbers $m_{k}$ and complex numbers $x_{k}, y_{k}$, where $k \in\{0, \ldots, n\}$, we observe that the convergence of the series $\sum_{k=0}^{\infty} m_{k}\left|x_{k}\right|^{p}$ and $\sum_{k=0}^{\infty} m_{k}\left|y_{k}\right|^{q}$ imply the convergence of the series $\sum_{k=0}^{\infty} m_{k}\left|x_{k}\right|^{p-1}\left|y_{k}\right|^{q-1}$.

Utilizing (3.7), we can state that

$$
\sum_{n=0}^{m}\left|a_{n}\right| k^{2 n} w^{n}(A) w^{n}(B) \leq \frac{\sum_{n=0}^{m}\left|a_{n}\right| k^{p n} w^{p n}(A) \sum_{n=0}^{m}\left|a_{n}\right| k^{q n} w^{q n}(B)}{\sum_{n=0}^{m}\left|a_{n}\right| k^{(p+q-2) n} w^{(p-1) n}(A) w^{(q-1) n}(B)}
$$

for any $m \in \mathbb{N}$ with $m \geq 1$.

This together with (3.4) provides

$$
w\left[\sum_{n=0}^{m} a_{n}(A B)^{n}\right] \leq \frac{\sum_{n=0}^{m}\left|a_{n}\right| k^{p n} w^{p n}(A) \sum_{n=0}^{m}\left|a_{n}\right| k^{q n} w^{q n}(B)}{\sum_{n=0}^{m}\left|a_{n}\right| k^{(p+q-2) n} w^{(p-1) n}(A) w^{(q-1) n}(B)}
$$

for any $m \in \mathbb{N}$ with $m \geq 1$.

Since all the series whose partial sums are involved in (3.8) are convergent, then by taking $n \rightarrow \infty$ in (3.8), we deduce the second inequality in (3.2).

Remark 1 If we take $p=q=2$ in the first inequality in (3.2), we have

$$
w^{2}(f(A B)) \leq f_{a}\left(k^{2} w^{2}(A)\right) f_{a}\left(k^{2} w^{2}(B)\right)
$$

provided $k^{2} w^{2}(A), k^{2} w^{2}(B)<R$.

Corollary 4 Let $f(z)=\sum_{n=0}^{\infty} a_{n} z^{n}$ be a function defined by power series with complex coefficients and convergent on the open disk $D(0, R) \subset \mathbb{C}, R>0$. Then for any $A, B \in B(H)$ with $2^{p} w^{p}(A), 2^{q} w^{q}(B)<R$, for $p>1, \frac{1}{p}+\frac{1}{q}=1$, we have the inequalities

$$
w(f(A B)) \leq \min \left\{N_{1}, N_{2}\right\}
$$

where

$$
N_{1}:=f_{a}^{1 / p}\left(2^{p} w^{p}(A)\right) f_{a}^{1 / q}\left(2^{q} w^{q}(B)\right)
$$


and

$$
N_{2}:=\frac{f_{a}\left(2^{p} w^{p}(A)\right) f_{A}\left(2^{q} w^{q}(B)\right)}{f_{a}\left(2^{(p+q-2)} w^{p-1}(A) w^{q-1}(B)\right)} .
$$

If $A, B \in B(H)$ are commutative with $2^{p / 2} w^{p}(A), 2^{q / 2} w^{q}(B)<R$, for $p>1, \frac{1}{p}+\frac{1}{q}=1$, then we have the inequalities

$$
w(f(A B)) \leq \min \left\{P_{1}, P_{2}\right\}
$$

where

$$
P_{1}:=f_{a}^{1 / p}\left(2^{p / 2} w^{p}(A)\right) f_{a}^{1 / q}\left(2^{q / 2} w^{q}(B)\right)
$$

and

$$
P_{2}:=\frac{f_{a}\left(2^{p / 2} w^{p}(A)\right) f_{a}\left(2^{q / 2} w^{q}(B)\right)}{f_{a}\left(2^{(p+q-2) / 2} w^{p-1}(A) w^{q-1}(B)\right)} .
$$

The proof of the inequality (3.10) follows by Theorem 5 since in this case, we can take $k=2$ in (3.1), while the inequality (3.11) follows by the commutative case, in which case we can take $k=\sqrt{2}$ in (3.1).

The case of commuting operators can be treated in a different way as well.

Proposition 1 Let $f(z)=\sum_{n=0}^{\infty} a_{n} z^{n}$ be a function defined by power series with complex coefficients and convergent on the open disk $D(0, R) \subset \mathbb{C}, R>0$. If $A, B \in B(H)$ are commutative with $w^{p}(A), w^{q}(B)<R$, for $p>1, \frac{1}{p}+\frac{1}{q}=1$, then we have the inequalities

$$
w(f(A B)) \leq 2 \min \left\{Q_{1}, Q_{2}\right\},
$$

where

$$
Q_{1}:=f_{a}^{1 / p}\left(w^{p}(A)\right) f_{a}^{1 / q}\left(w^{q}(B)\right)
$$

and

$$
Q_{2}:=\frac{f_{a}\left(w^{p}(A)\right) f_{a}\left(w^{q}(B)\right)}{f_{a}\left(w^{p-1}(A) w^{q-1}(B)\right)} .
$$

Proof Since $A, B \in B(H)$ are commutative, then for any $n \in \mathbb{N}$, the operators $A^{n}$ and $B^{n}$ are commutative and $A^{n} B^{n}=(A B)^{n}$.

Applying Theorem 5 for the commutative case, we have

$$
w\left((A B)^{n}\right)=w\left(A^{n} B^{n}\right) \leq 2 w\left(A^{n}\right) w\left(B^{n}\right) \leq 2 w^{n}(A) w^{n}(B)
$$

for any $n \in \mathbb{N}$.

Let $m \in \mathbb{N}$ with $m \geq 1$. We have, by the above inequality,

$$
w\left[\sum_{n=0}^{m} a_{n}(A B)^{n}\right] \leq \sum_{n=0}^{m}\left|a_{n}\right| w\left[(A B)^{n}\right] \leq 2 \sum_{n=0}^{m}\left|a_{n}\right| w^{n}(A) w^{n}(B) .
$$


Now, on making use of a similar approach to the one employed in the proof of Theorem 10 , we deduce the desired result (3.12).

Remark 2 If we take $p=q=2$ in the first inequality in (3.12), we get

$$
w^{2}(f(A B)) \leq 2 f_{a}\left(w^{2}(A)\right) f_{a}\left(w^{2}(B)\right)
$$

provided $w^{2}(A), w^{2}(B)<R$.

As pointed out in the introduction, the inequality

$$
w(A B) \leq c w(A)\|B\|
$$

holds for any two commuting operators $A, B \in B(H)$ and for some $c>1$. It is known that $1.064<c<1.169$; see $[6,7]$ and [8].

Proposition 2 Let $f(z)=\sum_{n=0}^{\infty} a_{n} z^{n}$ be a function defined by power series with complex coefficients and convergent on the open disk $D(0, R) \subset \mathbb{C}, R>0$. If $A, B \in B(H)$ are commutative with $w^{p}(A),\|B\|^{q}<R$, for $p>1, \frac{1}{p}+\frac{1}{q}=1$, then we have the inequalities

$$
w(f(A B)) \leq c \min \left\{Q_{1}, Q_{2}\right\}
$$

where

$$
S_{1}:=f_{a}^{1 / p}\left(w^{p}(A)\right) f_{a}^{1 / q}\left(\|B\|^{q}\right)
$$

and

$$
S_{2}:=\frac{f_{a}\left(w^{p}(A)\right) f_{a}\left(\|B\|^{q}\right)}{f_{a}\left(w^{p-1}(A)\|B\|^{q-1}\right)} .
$$

Moreover, if the operators $A$ and $B$ double commute, then the constant c can be taken to be 1 in (3.15).

Proof Applying the inequality (3.14) for the commuting operators $A^{n}$ and $B^{n}$ with $n \in \mathbb{N}$, we have

$$
w\left((A B)^{n}\right)=w\left(A^{n} B^{n}\right) \leq c w\left(A^{n}\right)\left\|B^{n}\right\| \leq c w^{n}(A)\|B\|^{n}
$$

for any $n \in \mathbb{N}$.

On making use of a similar argument as in the proof of Theorem 10, we deduce the desired inequality (3.15).

If the operators $A$ and $B$ double commute, then the operators $A^{n}$ and $B^{n}$ also double commute, and by Theorem 7 we deduce the second part of the proposition.

From a different perspective, we have the following result as well. 
Proposition 3 Let $f(z)=\sum_{n=0}^{\infty} a_{n} z^{n}$ be a function defined by power series with complex coefficients and convergent on the open disk $D(0, R) \subset \mathbb{C}, R>0$. If $A, B \in B(H)$ such that $\|A\|^{2},\|B\|^{2}<R$, then

$$
w(f(A B)) \leq\left\{\begin{array}{l}
f_{a}\left(\left\|\frac{A A^{*}+B^{*} B}{2}\right\|\right) \\
f_{a}\left(\left\|\frac{A A^{*}+B B^{*}}{2}\right\|\right)
\end{array} \leq \frac{f_{a}\left(\|A\|^{2}\right)+f_{a}\left(\|B\|^{2}\right)}{2} .\right.
$$

Proof We use the following two inequalities obtained by Kittaneh in [3]

$$
w(A B) \leq\left\{\begin{array}{l}
\left\|\frac{A A^{*}+B^{*} B}{2}\right\|, \\
\left\|\frac{A A^{*}+B B^{*}}{2}\right\|
\end{array}\right.
$$

for any $A, B \in B(H)$.

Let $n \in \mathbb{N}$. We have, by the above inequalities,

$$
w\left[(A B)^{n}\right] \leq w^{n}(A B) \leq\left\{\begin{array}{l}
\left\|\frac{A A^{*}+B^{*} B}{2}\right\|^{n} \\
\left\|\frac{A A^{*}+B B^{*}}{2}\right\|^{n}
\end{array} \leq \frac{1}{2}\left[\|A\|^{2 n}+\|B\|^{2 n}\right] .\right.
$$

The proof follows now as above and the details are omitted.

\section{Examples}

As some natural examples that are useful for applications, we can point out that if

$$
\begin{aligned}
& f(z)=\sum_{n=1}^{\infty} \frac{(-1)^{n}}{n} z^{n}=\ln \frac{1}{1+z}, \quad z \in D(0,1) ; \\
& g(z)=\sum_{n=0}^{\infty} \frac{(-1)^{n}}{(2 n) !} z^{2 n}=\cos z, \quad z \in \mathbb{C} ; \\
& h(z)=\sum_{n=0}^{\infty} \frac{(-1)^{n}}{(2 n+1) !} z^{2 n+1}=\sin z, \quad z \in \mathbb{C} ; \\
& l(z)=\sum_{n=0}^{\infty}(-1)^{n} z^{n}=\frac{1}{1+z}, \quad z \in D(0,1) ;
\end{aligned}
$$

then the corresponding functions constructed by the use of the absolute values of the coefficients are

$$
\begin{aligned}
& f_{A}(z)=\sum_{n=1}^{\infty} \frac{1}{n !} z^{n}=\ln \frac{1}{1-z}, \quad z \in D(0,1) ; \\
& g_{A}(z)=\sum_{n=0}^{\infty} \frac{1}{(2 n) !} z^{2 n}=\cosh z, \quad z \in \mathbb{C} ; \\
& h_{A}(z)=\sum_{n=0}^{\infty} \frac{1}{(2 n+1) !} z^{2 n+1}=\sinh z, \quad z \in \mathbb{C} ; \\
& l_{A}(z)=\sum_{n=0}^{\infty} z^{n}=\frac{1}{1-z}, \quad z \in D(0,1) .
\end{aligned}
$$


Other important examples of functions as power series representations with nonnegative coefficients are:

$$
\begin{aligned}
& \exp (z)=\sum_{n=0}^{\infty} \frac{1}{n !} z^{n}, \quad z \in \mathbb{C} ; \\
& \frac{1}{2} \ln \left(\frac{1+z}{1-z}\right)=\sum_{n=1}^{\infty} \frac{1}{2 n-1} z^{2 n-1}, \quad z \in D(0,1) ; \\
& \sin ^{-1}(z)=\sum_{n=0}^{\infty} \frac{\Gamma\left(n+\frac{1}{2}\right)}{\sqrt{\pi}(2 n+1) n !} z^{2 n+1}, \quad z \in D(0,1) ; \\
& \tanh ^{-1}(z)=\sum_{n=1}^{\infty} \frac{1}{2 n-1} z^{2 n-1}, \quad z \in D(0,1) ; \\
& { }_{2} F_{1}(\alpha, \beta, \gamma, z)=\sum_{n=0}^{\infty} \frac{\Gamma(n+\alpha) \Gamma(n+\beta) \Gamma(\gamma)}{n ! \Gamma(\alpha) \Gamma(\beta) \Gamma(n+\gamma)} z^{n}, \quad \alpha, \beta, \gamma>0, z \in D(0,1) ;
\end{aligned}
$$

where $\Gamma$ is a gamma function.

For any operator $T \in B(H)$ with $w(T)<1$, by making use of the inequality (2.1), we have the simple inequalities

$$
\begin{aligned}
& w\left[(I \pm T)^{-1}\right] \leq[1-w(T)]^{-1}, \\
& w\left[\ln (I \pm T)^{-1}\right] \leq \ln [1-w(T)]^{-1}, \\
& w\left[\sin ^{-1}(T)\right] \leq \sin ^{-1}[w(T)]
\end{aligned}
$$

and

$$
w\left[{ }_{2} F_{1}(\alpha, \beta, \gamma, T)\right] \leq{ }_{2} F_{1}(\alpha, \beta, \gamma, w(T)) .
$$

For any operator $T \in B(H)$, we also have

$$
\begin{aligned}
& w[\exp (T)] \leq \exp [w(T)], \\
& w[\sin (T)], w[\sinh (T)] \leq \sinh (w(T))
\end{aligned}
$$

and

$$
w[\cos (T)], w[\cosh (T)] \leq \cosh (w(T)) .
$$

Similar inequalities may be stated by employing the other results obtained for one or two operators. However, the details are left to the interested reader. 


\section{References}

1. Gustafson, KE, Rao, DKM: Numerical Range. Springer, New York (1997)

2. Kittaneh, F: A numerical radius inequality and an estimate for the numerical radius of the Frobenius companion matrix. Stud. Math. 158(1), 11-17 (2003)

3. Kittaneh, F: Numerical radius inequalities for Hilbert space operators. Stud. Math. 168(1), 73-80 (2005)

4. Dragomir, SS: Inequalities for the norm and the numerical radius of linear operators in Hilbert spaces. Demonstr. Math. XL(2), 411-417 (2007)

5. Holbrook, JAR: Multiplicative properties of the numerical radius in operator theory. J. Reine Angew. Math. 237, 166-174 (1969)

6. Davidson, KR, Holbrook, JAR: Numerical radii of zero-one matrices. Mich. Math. J. 35, 261-267 (1988)

7. Müller, V: The numerical radius of a commuting product. Mich. Math. J. 39, 255-260 (1988)

8. Okubo, K, Ando, T: Operator radii of commuting products. Proc. Am. Math. Soc. 56, 203-210 (1976)

9. Dragomir, SS, Sándor, J: Some generalisations of Cauchy-Buniakowski-Schwartz's inequality. Gaz. Mat. Metod. (Bucharest) 11, 104-109 (1990) (in Romanian)

10. Dragomir, SS: A survey on Cauchy-Bunyakovsky-Schwarz type discrete inequality. J. Inequal. Pure Appl. Math. 4(3), Article ID 63 (2003). Online http://www.emis.de/journals/JIPAM/article301.html?sid=30

doi:10.1186/1029-242X-2013-298

Cite this article as: Dragomir: Some numerical radius inequalities for power series of operators in Hilbert spaces. Journal of Inequalities and Applications 2013 2013:298.

\section{Submit your manuscript to a SpringerOpen ${ }^{\circ}$ journal and benefit from:}

- Convenient online submission

- Rigorous peer review

- Immediate publication on acceptance

- Open access: articles freely available online

- High visibility within the field

- Retaining the copyright to your article 